is already most appreciated in practice is the use of the instrument to control the annealing processes in connection with copper and its alloys. The whole history of the constitution and structure of the more important alloys of copper with one added element at a time has been worked out and embodied in what appear at first sight to be highly theoretical "equilibrium diagrams." These diagrams, however, enable us to understand the precise effect produced upon the constitution and structure of any of these alloys by thermal treatment. The structures resulting from exposure to certain temperatures, followed by either slow or rapid cooling, have been determined, as well as the particular properties of the alloys which correspond to these structures. An understanding of these diagrams therefore enables the manufacturer or user to treat his alloys at the proper temperatures, and to control the results with ease and certainty by examining a few specimens of the metal under the microscope and noting the type and the size or scale of the structure.

At the present moment the control of these processes is only satisfactorily available for those groups of alloys the constitution of which has been fully investigated, but this is so far the case only for binary alloys-i.e. those consisting of two metals only. The majority of industrial alloys are much more complex, and for these the theoretical guidance is not yet available, principally because the complete study of these complex systems is a matter of much greater difficulty than that of the simpler binary series. The fullest benefit of the microscope will therefore only become available for workers who deal with these complex alloys when the purely scientific investigations have covered this difficult ground; but meanwhile it is quite possible in practice to obtain empirical data as regards the best micro-structure and the treatment required to obtain it. Such data, although not of equal value with the more complete knowledge, form a useful temporary substitute.

These few indications of the present practical utility of the microscope in connection with engineering materials, while very far from covering the whole range of the subject, may perhaps be enough to show that, even with existing knowledge, the instrument is capable of rendering - and is, in fact, renderingthe greafest service to engineering and metallurgical practice. These fruits are already derived from little more than twenty years of metallographic investigation. For the future of this young science, therefore, the highest hopes appear to be well founded.

\section{WaLter ROSENHAIN.}

\section{THE YIELDING OF THE EARTH TO DISTURBING FORCES. ${ }^{1}$}

THE problem of determining how much the earth as a whole actually yields to the tidal disturbing forces of the sun and moon was definitely brought before scientific men by Lord Kelvin. He pointed out that, from observations of the tides of long period, it ought to be possible to obtain some definite information, and he urged the establishment of gravitational observatories fitted with instruments for detecting the lunar disturbance of gravity. However rigid the body of the earth may be, it necessarily yields a little to the deforming action of the sun and moon. This action produces two kinds of effect. In the first place, it alters the shape of the earth. If the earth were a perfect sphere, it would be drawn

1 Based on a paper by Prof. A. E. H. Love, F.R.S., read before the Royal Society on January $\mathrm{I}_{4}$.

$$
\text { No. } 206 \mathrm{I} \text {, VOL. } 80]
$$

out by the attraction of the moon, for instance, into a prolate ellipsoid of revolution. The actual earth, of a shape that is nearly spherical but presents certain inequalities, acquires under the action of the moon a slight additional inequality of figure, of the same type as that which answers to elongation in the direction of the long axis of the ellipsoid and flattening round the parts remote from that axis. As the moon moves relatively to the earth, the long axis of the ellipsoid moves about in the earth, so that a corporeal tide is raised in the earth. Besides raising a corporeal tide, the action of the moon alters the attraction of the earth. If the change of external shape only is taken into account, the alteration of the attraction consists of the added attraction, due to the protuberances at the ends of the long axis of the ellipsoid, coupled with the loss of attraction, due to the flattening round the parts remote from these ends. But, since the material of which the earth is made up is not homogeneous, a similar effect is produced by the elongation and flattening of the surfaces of equal density, and, since the material is not absolutely incompressible, the density must be in some parts increased and in others diminished, owing to the attraction of the moon being different in different parts. The alteration of the earth's attraction by the action of the moon is therefore of a somewhat complex character. The effects produced by the action of the sun are similar to those produced by the action of the moon.

Many attempts have been made to measure the changes of level that are due to the tidal disturbing forces of the sun and moon. In the majority of such attempts, instruments of the horizontal pendulum type have been used. The displacement of a horizontal pendulum that would be produced by the attraction of the moon, or the sun, if the earth were absolutely rigid, is known, for the attractions of the moon and sun are known. In the actual case, owing to the yielding of the earth, all we can hope to determine by observations of the tides or of the displacement of horizontal pendulums is a relative change of level, and to measure this is far from easy. The effect to be measured is extremely minute, and it is liable to be obscured, or even disguised altogether, by the effects of air currents and of changes of temperature. Recently Dr. O. Hecker, of Potsdam, has succeeded in overcoming the experimental difficulties. By setting up two horizontal pendulums in an underground chamber, and observing their behaviour during a protracted period, he was able to show that the effect of the moon, in particular, is perfectly definite, that in phase it follows very closely the motion of the moon, and that in amount it is almost exactly two-thirds of what it would be if the earth were absolutely rigid.

Hecker's result confirms decisively the results which had been found with much less perfect experimental means by previous observers. It leaves no shadow of doubt of the actuality of a corporeal tide produced by the moon. It accords also with those results, deduced from observations of fortnightly tides, which were used by Lord Kelvin in his famous estimate of the rigidity of the earth. This estimate was obtained by working out mathematically the change of shape that would be produced by the attraction of an external body, such as the moon, in a solid elastic globe, of the same size and mass as the earth, if the material of which it is made were homogeneous and absolutely incompressible. When these simplifying assumptions are made, the change of attraction is calculable in terms of the change of shape, and the measurement of the relative change of level leads easily to the determination of the absolute change of 
level. If with these simplifying assumptions there is combined the observed fact that the relative change of level is two-thirds of what it would be if the earth were absolutely rigid, it is found, as Lord Kelvin did in effect find, that the calculated rise and fall of the surface is one-third of what it would be if the earth were made of homogeneous incompressible fluid, and the calculated change of its attraction due to the sun, or moon, is one-half of the tide-generating force of the sun, or moon. The rigidity which the material, supposed homogeneous and incompressible, would need to have in order that the two numbers may have the calculated values, $\frac{1}{3}$ and $\frac{1}{2}$, is about the same as the rigidity of steel. Both the numbers $\frac{1}{3}$ and $\frac{1}{2}$, which are thus calculated are inferred, partly from a result of observation, and partly from the subsidiary hypotheses of homogeneity and incompressibility. If these hypotheses are discarded, all that can be inferred from observations of fortnightly tides and horizontal pendulums is a single equation connecting two numbers. The number which in the special case is $\frac{1}{3}$ is in general conveniently written as $\frac{2}{5} \mathrm{~h}$, and the number which in the special case is $\frac{1}{2}$ may be called $k$. The observations in question concur in leading to the equation $h-k=\frac{1}{3}$. (In the special case $\frac{5}{6}-\frac{1}{2}=\frac{1}{3}$.)

It was first suggested by Prof. Simon Newcomb that the length of the earth's free period of nutation, usually called the "Chandler period," may be an independent index of the yielding of the earth to small forces. It has long been known that if the earth were absolutely rigid this period would be about 306 days. A free nutation of the earth would be manifested by periodic changes of latitude of places on its surface. Small variations of latitude have long been known to exist, but all efforts of astronomers to detect a period of 306 days in these variations failed. It was announced by Dr. S. C. Chandler, in $189 \mathrm{I}$, that the variations are roughly periodic, but that the period is really 427 days instead of 306 . Newcomb pointed out that the lengthening of the period must be due to a yielding of the earth. At any instant the earth is rotating about an axis which does not quite coincide with a principal axis. A solid globe would be deformed by rotation into an oblate spheroid in the same way as a fluid one, but not so much. The inequality of the so-called "centrifugal force," due to the deviation of the instantaneous axis from a principal axis, produces a slight deformation of the surface, accompanied by a slight alteration of the attraction, and these effects can be specified by means of the same two numbers $h$ and $k$ as are required to express the effects of tidal disturbing forces. Mr. S. S. Hough, H.M.'s Astronomer at the Cape of Good Hope, calculated, in 1896 , the lengthening of the period in the case of a solid elastic globe of homogeneous incompressible material. The problem has recently been discussed in a more general way by Prof. G. Herglotz, who was able to dispense with the hypothesis of homogeneity. A review of the theory, as presented by Herglotz, shows that it is possible to dispense with the hypothesis of incompressibility also, and that the lengthening of the period depends upon the number $k$, and not upon the number $h$. The number $k$ is found to be expressible in terms of the two periods ( 306 and 427 days), the ellipticity and mean radius of the surface, the angular velocity of rotation, and the mean value of gravity at the surface. This number is therefore known. Its value is found to be about $\frac{4}{15}$. The result that $k=\frac{4}{15}$ means that the alteration in the attraction of the earth on account of the distortion produced in it by the sun or moon is actually about four-fifteenths

No. $206 \mathrm{r}$, vol. 80$]$ of the tide-generating force of the sun or moon. This result does not depend upon any hypothesis as to the homogeneity or incompressibility of the material. The only assumptions that are used in obtaining it are the assumption that an equilibrium theory is applicable to the forces in question, and the assumption, commonly made in the theory of the figure of the earth since the time of Laplace, viz. that the surfaces of equal density within the earth are maintained in ellipsoidal shapes by the rotation. The result does not depend upon the special hypothetical law of density which Laplace introduced. Any law of density which satisfies the ordinary laws of hydrostatics will suffice. ${ }^{1}$

When the result expressed by the equation $k=\frac{4}{15}$ is combined with the result of observations of the tides and horizontal pendulums $\left(h-k=\frac{1}{3}\right)$, it is found that $h=\frac{3}{5}$. This result means that the surface of the earth actually yields to the tidal deforming influence of the sun and moon by six-twenty-fifths of the amount by which it would yield if the earth were made of homogeneous incompressible fluid. The number $\frac{6}{25}$ takes the place of Lord Kelvin's number ${ }_{3}^{1}$.

The result that the earth actually yields a good deal less than Lord Kelvin supposed it to do suggests that it is decidedly more rigid than he estimated it to be. There are, however, many difficulties in the way of a more precise estimate, the chief being the heterogeneity of the material. If this fact is disregarded, and the simplifying assumption of homogeneity is made, it appears to be impossible to satisfy both the equations $h=\frac{3}{5}$ and $k=\frac{4}{1} \frac{4}{5}$. An additional difficulty arises from the compressibility of the material, but, although this cannot be met directly, it is not very serious, because the general effect of compressibility must almost certainly be that any estimate of rigidity based on the simplifying assumption of incompressibility is under the mark. A possible method of procedure is to assume the earth to consist of a central nucleus of incompressible material of one density and rigidity, enclosed in a shell of incompressible material of a smaller density and a different rigidity, in the manner advocated by Prof. E. Wiechert, who regards the earth as made up of an iron core enclosed in a rocky shell. This method was developed by Dr. W. Schweydar, who found that, with the densities proposed by Wiechert, the rigidity of the core would have to be nearly three times that of steel, and the rigidity of the shell about one-eighth of that of steel. The possibility of a comparatively small rigidity in the enclosing shell suggests that there may be within it, or between it and the core, a layer of molten rock, devoid of rigidity, such as has sometimes been invoked in connection with the explanation of seismic and volcanic phenomena. This hypothesis is found, when tested mathematically, to require much too great rigidities both of the core and of the outer part of the shell. It appears, however, to be quite possible that the earth may consist of a very dense and very rigid core enclosed in, and connected by solid matter with, a lighter shell or crust, the greater part of which is solid and of a rigidity comparable with that of granite (about one-third of that of steel), the shell being honeycombed with hollow spaces containing molten matter. But it seems to be impossible that the molten matter should form a continuous layer separating the outer portions of the earth's body from the inner portions.

1 Since the paper was written and sent in to the Royal Society, Prof. Lar mor has shown that the result is independent of the supposed ellipsoidal shape of the surfaces of equal density. It is therefore established, quite generally, for any constitution of the earth which would admit of the application of an equilibrium theory to forces of the type in question. It is practically certain that the actual constitution is such that a theory of this kind can be applied. 